\title{
Quantifying The Human-Robot Interaction Forces Between A Lower Limb Exoskeleton And Healthy Users
}

\author{
Ashish Rathore ${ }^{1}$, Matthew Wilcox ${ }^{1}$, Dafne Zuleima Morgado Ramirez ${ }^{2}$, Rui Loureiro ${ }^{1}$, Member, \\ IEEE and Tom Carlson ${ }^{1}$, Member, IEEE
}

\begin{abstract}
To counter the many disadvantages of prolonged wheelchair use, patients with spinal cord injuries (SCI) are beginning to turn towards robotic exoskeletons. However, we are currently unaware of the magnitude and distribution of forces acting between the user and the exoskeleton. This is a critical issue, as SCI patients have an increased susceptibility to skin lesions and pressure ulcer development. Therefore, we developed a real-time force measuring apparatus, which was placed at the physical human-robot interface (pHRI) of a lower limb robotic exoskeleton. Experiments captured the dynamics of these interaction forces whilst the participants performed a range of typical stepping actions. Our results indicate that peak forces occurred at the anterior aspect of both the left and right legs, areas that are particularly prone to pressure ulcer development. A significant difference was also found between the average force experienced at the anterior and posterior sensors of the right thigh during the swing phase for different movement primitives. These results call for the integration of instrumented straps as standard in lower limb exoskeletons. They also highlight the potential of such straps to be used as an alternative/complementary interface for the high-level control of lower limb exoskeletons in some patient groups.
\end{abstract}

\section{INTRODUCTION}

The loss of sensorimotor control of the lower limbs is a hallmark of spinal cord injury (SCI). Wheelchairs are therefore used by patients in order to regain a degree of autonomy, and explore and interact with their environment. However, prolonged wheelchair use is associated with an array of complications, including shoulder pain, the profound loss of bone mineral density, and pressure ulcer development $[1,2]$. Moreover, wheelchair use is impractical for dealing with certain environmental situations, such as reaching heights (e.g. shelves) and climbing stairs. Therefore, patients are beginning to turn to robotic exoskeletons [3], an emerging technology which has the potential to transform the lives of patients psychologically, socially and physically.

There is both a cognitive and a physical coupling between the user and the exoskeleton. The latter involves multiple points of human-robot contact, at which a net flux of power generated by the exoskeleton is transferred to the viscoelastic soft tissues of the patient [3]. This transfer of

A. Rathore, M. Wilcox, R. Loureiro and T. Carlson are with Aspire CREATe, the Centre for Rehabilitation Engineering and Assistive Technology, Royal National Orthopedic Hospital, University College London (phone: +44 208385 3048; fax: +44 208954 8560; email: t.carlson@ucl.ac.uk).

D. Z. Morgado Ramirez is with the Institute of Orthopedics and Musculoskeletal Science, Royal National Orthopedic Hospital, University College London. power is distributed through two interfaces: connection cuffs (soft belts) and orthoses (plastic braces against which the leg is supported) [4]. Furthermore, the complexity of human joint kinematics is almost impossible to emulate exactly in robotic design; joint movement is influenced by a variety of internal structures (i.e., ligaments and tendons) and the inherent migration of the joint centre during movement [5]. As a consequence, the axes of joint rotation between the user and the exoskeleton are micro-misaligned, which generates potentially harmful interaction forces, such as shear forces at the physical human-robot interface pHRI [5].

Further studies investigating the changes in physiological metrics during exoskeleton use and on the safety of these devices need to be performed [3]. Currently, the strapping protocol used by trained therapists when fastening a user into an exoskeleton is largely based on heuristic personal experience. This is a critical issue as SCI patients are particularly vulnerable to skin lesions and pressure ulcer development due to a combination of impaired sensation, and physiological changes in denervated skin, which prevent efficient wound healing. To date, there have been few studies that investigate the interaction forces at the pHRI, these include using mathematical modeling [7], direct measurement using load cells [8] and opto-electronic sensors [9]. However, the accuracy of the mathematical model is difficult to measure due to the complex interaction dynamics presented by the viscoelastic soft tissues and elastic cuffs. Moreover, the single point sensors used in these studies do not provide information on the distribution of force across the human-exoskeleton interface (cuffs and orthoses), and we therefore only know of the pressures acting at one particular point of the interface. Although an array of load cells could have been constructed to measure a multiple number of contact points, this is both complex to calibrate and costly.

One noteworthy study developed pressure pads that were used to record pressure maps for one healthy participant and one SCI patient [6]. However, integrating force sensitive resistors (FSRs) into a flexible pressure-distributing pad may cause undue bending of the FSRs during applied loads, which will likely affect the voltage output and hence the accuracy of their results. By contrast, in this paper we identified 16 key locations to monitor and obtained data from 10 healthy participants, which yielded statistically significant results. We used healthy participants so as to avoid the complications of impaired sensation at this stage.

Hence, this study presents the development of an alternative real-time force-monitoring apparatus using FSRs installed at the pHRI. We also present an experimental 
Figure 1. The REX exoskeleton (Rex Bionics), instrumented with four force sensitve resistors (FSRs) at each of the four physical human-robot interfaces (pHRIs) on the lower limbs.

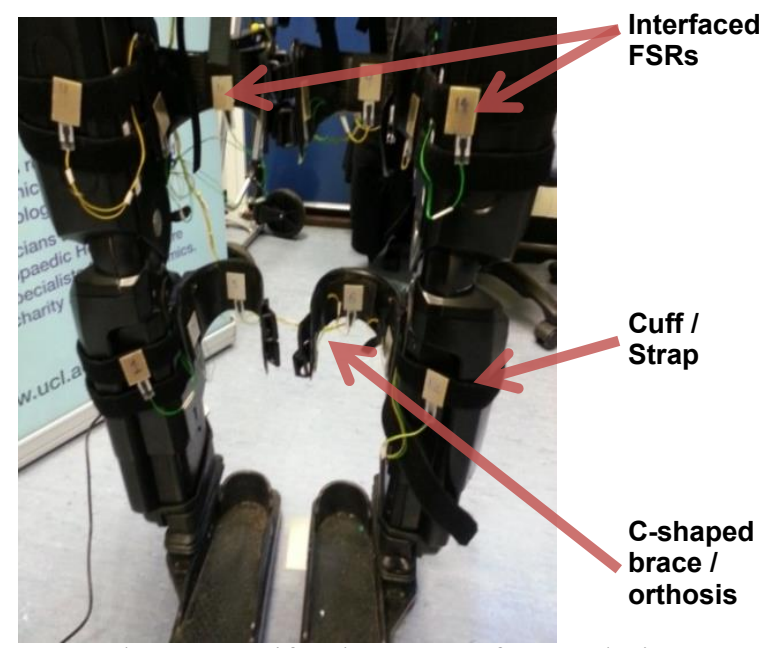

protocol to quantify the pHRI forces during a range of exoskeleton movement primitives.

\section{MATERIALS AND METHODS}

The Interlink Electronics force sensitive resistor (FSR) 400 was selected for our application, due to its low cost, reliability and versatility. It has a large and appropriate sensitivity range for our application $(0.1 \mathrm{~N}-100 \mathrm{~N})$ and has a small sensing area $(5.1 \mathrm{~mm}$ diameter $)$, which allows for force measurements over small contact points and minimizes bending effects of the cuff/strap. A robotic exoskeleton, REX Personal (Rex Bionics, New Zealand), which is controlled by an intuitive joystick interface, was used for the experiments.

The experimental apparatus is set up as shown in Fig. 1. Four sensors were placed at each of the four c-shaped braces/orthosis ( 2 thigh braces and 2 leg braces): medially, laterally and posteriorly on the brace, and one anteriorly by interfacing with the strap (16 FSRs in total). With this arrangement we were able to make the following observations:

1. Shifts in interaction forces during gait or other movement primitives.

2. Regions of principal and insignificant forces.

3. Force distribution across the whole pHRI.

4. The average force experienced by the limb segment in these four directions.

For the purposes of force data acquisition we used an Arduino microcontroller circuit board (Arduino Mega 2560, Italy) and built a graphical user interface (GUI) in Matlab (MathWorks, USA).

Each sensor was interfaced with a plastic Velcro backing and individually calibrated so as to minimize any systematic error in the measurements. The calibration was performed using a mechanical testing machine (Zwick Roell Z005, Germany), equipped with a $5 \mathrm{kN}$ load cell. A flat, rigid stainless steel indenter, whose diameter matched the area of the FSR sensing area, was applied over the Velcro at the position that corresponded to the sensing area of the underlying FSR. For each sensor, a ramped load between 1 and $100 \mathrm{~N}$ was applied at a speed of $1 \mathrm{~mm} / \mathrm{minute}$ ( 3 repeats) For each sensor, a force-deformation profile was recorded simultaneously with the voltage response of the sensor. The force vs voltage output data was then plotted using Matlab and fitted with a first-order exponential equation, with an average $\mathrm{R}^{2}$ value of 0.9373 for the 16 sensors.

Ethical approval for this study was obtained from University College London Research Ethics Committee (6859/001). The inclusion criteria were: physically and cognitively healthy adults between the ages of 18 and 65 . Volunteers were excluded if they had any pathology affecting gait.

Each participant was asked to wear the exoskeleton and the experimenter helped them to fasten the cuffs to a tension that the participant deemed comfortable. A period of five minutes was given to each participant to experience the movement primitives of the exoskeleton. After this, data was acquired whilst the participants performed two steps forward (a full gait cycle). This protocol was repeated again but this time taking two steps backward and then finally taking two left sidesteps. The participant repeated these protocols three times.

The gait cycle of REX does not emulate the natural gait of humans [10]. Therefore, a series of triggers were also developed in Matlab so that the force data could be related to the kinematics of the exoskeleton.

\section{RESULTS}

Data was recorded from 10 subjects and Fig. 2 illustrates a typical plot from a single sensor (left thigh, anterior) for one gait cycle. Participant demographics are outlined in Table 1

TABLE I. PARTICIPANT DEMOGRAPHICS.

\begin{tabular}{cc}
\hline Variables & Range \\
\hline Gender & Male $=8$, Female $=2$ \\
Age & $18-60$ years \\
Weight & $52-89 \mathrm{~kg}$ \\
Height & $164-186 \mathrm{~cm}$ \\
\hline
\end{tabular}

Forces acting anteriorly (across the exoskeleton strap) are higher in magnitude than those acting on the sensors that have been interfaced with the exoskeleton orthosis, as can be seen in Fig. 3. For example, the median peak force $(22.58 \mathrm{~N})$

Figure 2. Force data acquisition over one full gait cycle for the anterior force sensor of the left thigh for one of the participants

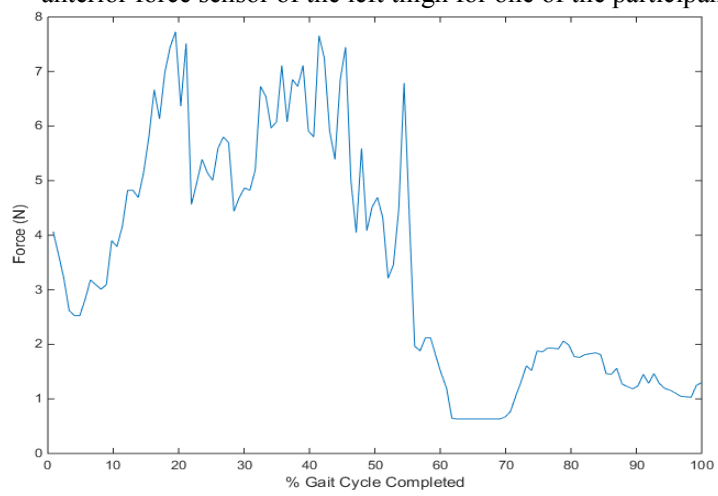


of the anterior sensor for the right thigh when taking a step forward is significantly higher than the median peak force of the medial $(1.91 \mathrm{~N})$, posterior $(4.83 \mathrm{~N})$ and lateral $(1.46 \mathrm{~N})$ sensors of the right thigh. The statistical significance of these results was determined using a (non-parametric) Friedman test $(\chi 2=19.56, \mathrm{p}<0.001)$ followed by pairwise comparisons with a Bonferroni correction for multiple comparisons $(\mathrm{p}<0.001, \mathrm{p}=0.034$ and $\mathrm{p}=0.002$, respectively). The exception to this is the anterior sensor of the left thigh, whose peak force is not significantly different compared to the medial, posterior and lateral sensors of the left thigh when walking forward, backward or to the side.

Only 5 of the 16 sensors interfaced with the exoskeleton showed a significant difference between the peak force at the pHRI $(p<0.005)$ for all three of the different movement primitives. In particular, the peak forces observed at the anterior aspect of the right and left legs showed a significant difference for the 3 different movement primitives according to a Friedman test; $\chi^{2}(2)=10.4, p=0.006$ and $\chi^{2}(2)=15.2$, $\mathrm{p}<0.002$, respectively (Fig. 3 ).

The peak force was found to increase from $21.37 \pm 7.67 \mathrm{~N}$ during forward walking to $24.45 \pm 12.06 \mathrm{~N}$ during backward walking and then to $29.88 \pm 10.05 \mathrm{~N}$ during left sidestepping for the right leg. The left leg showed an increase in peak force from $10.54 \pm 3.16 \mathrm{~N}$ to $14.69 \pm 6.17 \mathrm{~N}$ to $15.65 \pm 4.08 \mathrm{~N}$. Although peak forces differed as the user performed each of the different movement primitives, the difference was only significant between forward walking and sidestepping in the right leg $(p=0.005)$, whereas for the left leg, the peak force was significantly different between forward and backward walking $(p=0.005)$ and between forward walking and sidestepping $(\mathrm{p}=0.001)$.

A significant difference in force between forward $(2.14 \pm 1.27 \mathrm{~N})$ and backward $(7.16 \pm 0.64 \mathrm{~N})$ walking was only found at the anterior sensor of the right thigh during the swing phase of these movements $(p=0.002)$. A significant difference was found for forward and backward walking $(3.72 \pm 0.68 \mathrm{~N}$ and $0.298 \pm 0.14, \mathrm{p}=0.001)$, and forward and sidestepping $(3.72 \pm 0.68 \mathrm{~N}$ and $0.48 \pm 0.18 \mathrm{~N}, \mathrm{p}=0.005)$ for the posterior sensor of the right thigh during the swing phase of these exoskeleton movement primitives. Moreover, Fig. 4 demonstrates that the anterior and posterior sensors for the right thigh show a statistically significant difference in force during the swing phase for each of the three movement primitives.

\section{DISCUSSION}

Currently, the strapping protocol used by trained therapists when fastening a user into an exoskeleton is largely based on heuristic personal experience. It would therefore be advantageous for the therapist to be given realtime feedback about the interaction forces during the initial strapping procedure, as our developed apparatus does. Moreover, we found that five of the sensors displayed significant differences between the peak forces recorded at each sensor, whilst performing typical activities. This shows that the potential risk of skin lesion and pressure ulcer development at certain areas of the pHRI as a result of excessive force, is not simply dependent on the initial fastening of the cuff. Instead, it is also dependent on the type of activity being carried out by the exoskeleton.

The anterior aspect of the leg is particularly important because human tolerance to pressure and the risk of pressure ulcer development is dependent on the position of contact between the user and the environment $[11,12]$. This contact is enhanced at areas of bony prominences, such as at the anterior tibial aspect of the leg, where the soft tissues are more easily compressed between the exoskeleton cuff and the rigid underlying bone [12]. Moreover, the use of cuffs/straps as the physical interface between the user and exoskeleton could yield a greater risk of skin lesion and pressure ulcer development than the use of orthoses/braces as they have been shown to constrict the soft tissues and underlying capillaries, create frictional forces as they slide against the user's skin and/or clothes and can fold the user's skin $[13,14]$.

In this study, we have seen that the peak force

Figure 4. Boxplots for the average force across participants for the different movement primitives during the swing of the right limb for the anterior and posterior sensors of the right thigh. $*$ significant difference $(\mathrm{p}<0.05)$.

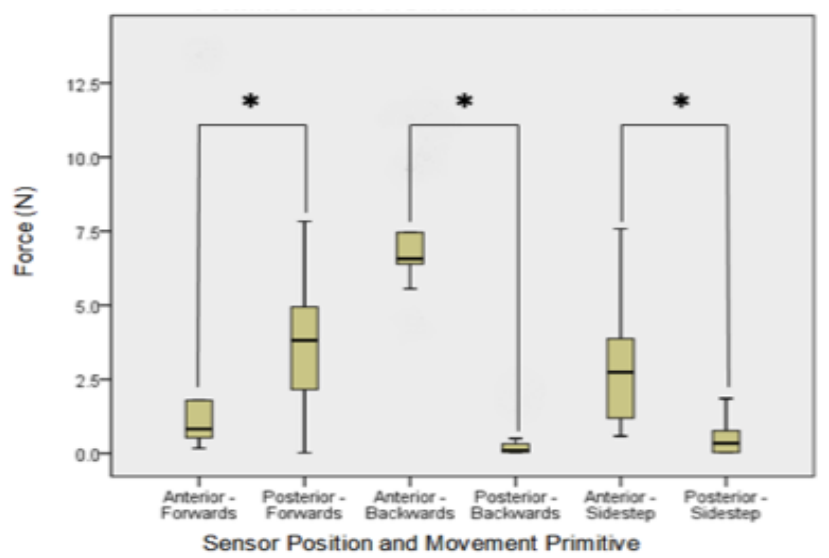

Figure 3. Average peak force for sensors across different limb segments when walking forward (leftmost plot), backward (centre plot) and to the side (rightmost plot). The asterisks represent statistically significant differences $(\mathrm{p}<0.005)$
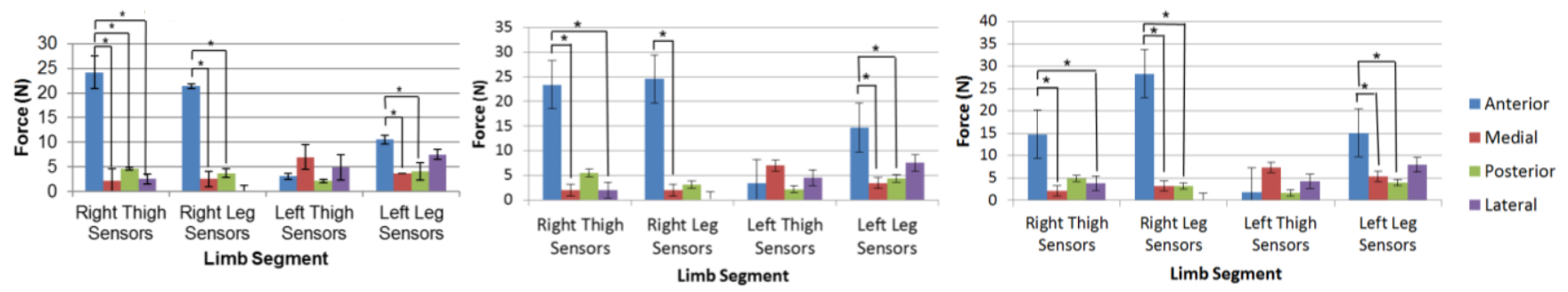
experienced at the anterior aspect of the right leg can increase by $8.51 \mathrm{~N}$ and for the left leg can increase by $5.11 \mathrm{~N}$ depending on the actual movement carried out. Consequently, we recommend that the straps of the exoskeleton be adjusted to accommodate for this significant change.

The forces we have measured in this study could potentially be attributed to a combination of voluntary and involuntary muscular contraction of the participant and not solely due to the exoskeleton. Such muscular activity could arise due to co-contraction [4], hesitance or anticipation. Therefore, we have already started to investigate EMG activity in healthy participants to assess whether the interaction forces observed in this study are (partially) due to the exoskeleton or muscular activity of the participant.

In the future it would be beneficial to develop "smart straps" that can automatically regulate the applied force to alleviate this challenge. However, the peak forces at the leg remain under the pressure pain threshold obtained by Lee et al. [15] at the mid-tibial crest $(0.45 \mathrm{MPa}$ - normal force of $50.9 \mathrm{~N})$. The use of this value as a threshold for our application should be used carefully as it was determined by calibrating the force sensors with a metal rigid indenter and with tests performed by healthy volunteers. Smart straps could integrate calibrated sensors for not only normal but also shear forces being applied through a non-rigid strap to SCI patients. Moreover, the prolonged application of forces below any pain threshold has nevertheless been shown to cause pathological morphological and biochemical changes even in healthy subjects [16].

\section{CONCLUSIONS}

A real-time, cost-effective force-monitoring device has been successfully developed and interfaced on a commercially available lower limb robotic exoskeleton. This study characterized the variation in force magnitude and distribution across the pHRI for a REX Exoskeleton during commonly used movement primitives.

We have shown that at the anterior aspect of the right and left legs-clinically important areas with regards to skin lesion development-peak forces differ significantly, depending on the movement primitive that is being executed. We recommend that the therapists using lower limb exoskeletons take these observations into account as they initially fasten the user into the device.

The study found no significant difference between the time-averaged forces for particular sensors during different movement primitives. However, the forces that occur during the swing phase of the exoskeleton when walking forward, backward or sideways differed significantly for the anterior and posterior sensors placed at the right thigh. Moreover, the average force at the anterior and posterior sensors of the right thigh during the swing phase of these different movement primitives was found to be significantly different. This information could be used in the development of a control algorithm for the exoskeleton in which user intention could be conveyed through interaction force. This study also highlights the benefits that "smart straps", able to regulate tension, could bring to the patient.

\section{ACKNOWLEDGMENT}

We are very grateful to REX Bionics for the provision of equipment and technical support throughout the project.

\section{REFERENCES}

[1] J. Carver, A. Ganus, J. M. Ivey, T. Plummer, and A. Eubank, "The impact of mobility assistive technology devices on participation for individuals with disabilities.," Disabil. Rehabil. Assist. Technol., pp. $1-10,2015$.

[2] K. A. M. Samuelsson, H. Tropp, and B. Gerdle, "Shoulder pain and its consequences in paraplegic spinal cord-injured, wheelchair users.," Spinal Cord, vol. 42, no. 1, pp. 41-46, Jan. 2004.

[3] A. M. Dollar and H. Herr, "Lower extremity exoskeletons and active orthoses: Challenges and state-of-the-art," IEEE Trans. Robot., vol. 24, no. 1, pp. 144-158, 2008.

[4] S. M. M. de Rossi, N. Vitiello, T. Lenzi, R. Ronsse, B. Koopman, A. Persichetti, F. Vecchi, A. J. Ijspeert, H. van der Kooij, and M. C. Carrozza, "Sensing pressure distribution on a lower-limb exoskeleton physical human-machine interface," Sensors, vol. 11, no. 1, pp. $207-$ 227, 2011.

[5] J. L. Pons, Wearable Robots: Biomechatronic Exoskeletons. 2008.

[6] J. Tamez-Duque, R. Cobian-Ugalde, A. Kilicarslan, A. Venkatakrishnan, R. Soto, and J. L. Contreras-Vidal, "Real-time strap pressure sensor system for powered exoskeletons," Sensors (Switzerland), vol. 15, no. 2, pp. 4550-4563, 2015.

[7] A. Schiele, "An explicit model to predict and interpret constraint force creation in phri with exoskeletons," in Robotics and Automation, 2008. ICRA 2008. IEEE International Conference on, 2008, pp. 13241330 .

[8] J. M. Hidler and A. E. Wall, "Alterations in muscle activation patterns during robotic-assisted walking," Clin. Biomech., vol. 20, no. 2, pp. 184-193, 2005.

[9] M. Donati, N. Vitiello, S. M. M. de Rossi, T. Lenzi, S. Crea, A. Persichetti, F. Giovacchini, B. Koopman, J. Podobnik, M. Munih, and M. C. Carrozza, "A flexible sensor technology for the distributed measurement of interaction pressure," Sensors (Switzerland), vol. 13, no. 1. pp. 1021-1045, 2013.

[10] G. Barbareschi, R. Richards, M. Thornton, T. Carlson, and C. Holloway, "Statically vs dynamically balanced gait: Analysis of a robotic exoskeleton compared with a human," in Proc of the Annu. Int. Conf. of the IEEE Engineering in Medicine and Biology Society, Millan, 2015, pp. 6728-6731.

[11] D. Bluestein and A. Javaheri, "Pressure ulcers: Prevention, evaluation, and management," American Family Physician, vol. 78, no. 10. 2008.

[12] M. J. Bass and L. G. Phillips, "Pressure sores.," Curr. Probl. Surg., vol. 44, no. 2, pp. 101-143, Feb. 2007.

[13] C. a Cushing and L. G. Phillips, "Evidence-based medicine: pressure sores.," Plast. Reconstr. Surg., vol. 132, no. 6, pp. 1720-32, 2013.

[14] J. R. Lowe, "Skin Integrity in Critically Ill Obese Patients," Critical Care Nursing Clinics of North America, vol. 21, no. 3. pp. 311-322, 2009.

[15] W. C. Lee, M. Zhang, and A. F. Mak, "Regional differences in pain threshold and tolerance of the transtibial residual limb: including the effects of age and interface material.," Arch. Phys. Med. Rehabil., vol. 86, no. 4, pp. 641-649, Apr. 2005.

[16] O. Stojadinovic, J. Minkiewicz, A. Sawaya, J. W. Bourne, P. Torzilli, J. P. de Rivero Vaccari, W. D. Dietrich, R. W. Keane, and M. TomicCanic, "Deep tissue injury in development of pressure ulcers: a decrease of inflammasome activation and changes in human skin morphology in response to aging and mechanical load.," PLoS One, vol. 8 , no. 8 , p. e69223, 2013. 